\title{
Prevalence of vitamin D deficiency and its associated factors among rheumatoid arthritis patients managed in a rheumatology unit of a tertiary care hospital in Sri Lanka
}

\author{
Authors: Sethuge Sanjeeva Chaminda Silva, ${ }^{\mathrm{A}}$ Gunendrika Kathurirathne, ${ }^{\mathrm{A}}$ Buddhika Mahesh, ${ }^{\mathrm{B}}$ Janalini Sashikaran ${ }^{\mathrm{A}}$ \\ and Kumari Jayasiri ${ }^{\mathrm{C}}$
}

\section{Introduction}

Prevalence of unrecognised vitamin D deficiency can be high among rheumatoid arthritis (RA) patients. Lack of mobility among these patients prevents them receiving adequate sun exposure. Low levels of vitamin D could potentially cause a higher disease burden and disease activity.

\section{Materials and methods}

A descriptive cross-sectional study was done among patients with RA with a calculated sample size of 137. Being diagnosed according to American College of Rheumatology (ACR) - European League Against Rheumatism (EULAR) criteria and availability of serum vitamin D level were among the inclusion criteria. Patients with disabilities due to causes other than RA were excluded. All patients satisfying the eligibility criteria were invited to be recruited. A data extraction sheet was utilised. Data was collected by investigators. The associations were evaluated with Chi square test and Spearman correlation coefficient at a significance level of $5 \%$. Ethics approval was obtained from the ethics committee of National Hospital of Sri Lanka (NHSL).

\section{Results and discussion}

The response rate was $92 \%$. The median (interquartile range (IQR)) age of participants was 56.5 (49.0 to 64.25). Among participants, the majority $(n=117,92.9 \%)$ were females. Only $11.1 \%(n=14)$ had normal vitamin $D$ levels. The insufficient and deficient categories comprised of $38.1 \%(n=48)$ and $50.8 \%(n=64)$. The commonest symptoms included; joint pain ( $n=101,80.2 \%)$, fatigue $(n=84$, $66.7 \%$ ) and muscle pain ( $n=78,61.9 \%)$. Deficiency or insufficiency was lowest in the occupation category of 'agricultural and labourer' (37.5\%) while 100\% in many indoor-occupied categories and among Muslims. 'Deficiency or insufficiency' was significantly associated with muscle pain ( $p=0.001)$ but not with Clinical

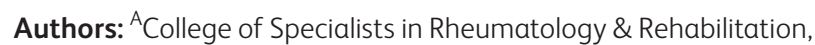

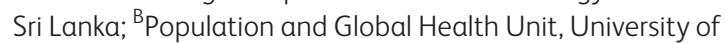
Melbourne, Australia; ${ }^{C}$ National Hospital of Sri Lanka, Sri Lanka
Disease Activity Index (CDAI) $(p=0.896)$, fatigue $(p=0.549)$ or joint pain $(p=0.735)$.

\section{Conclusions}

Vitamin D 'deficiency or insufficiency' is common among patients with RA and commoner in the sub-categories with muscle pain and with restricted sun exposure. More research must be promoted in this regard.

\section{Conflict of interest statement}

None declared. 